\title{
Time-Hopping Multicarrier Code-Division Multiple Access
}

\author{
Lie-Liang Yang, Senior Member, IEEE
}

\begin{abstract}
A time-hopping multicarrier code-division multipleaccess (TH/MC-CDMA) scheme is proposed and investigated. In the proposed TH/MC-CDMA, each information symbol is transmitted by a number of time-domain pulses with each time-domain pulse modulating a subcarrier. The transmitted information at the receiver is extracted from one of the, say, $M$ possible time-slot positions, i.e., assuming that $M$-ary pulse-position modulation is employed. Specifically, in this paper, we concentrate on the scenarios such as system design, power spectral density (PSD) and single-user-based signal detection. The error performance of the TH/MC-CDMA system is investigated when each subcarrier signal experiences flat Nakagami- $m$ fading in addition to additive white Gaussian noise. According to our analysis and results, it can be shown that the TH/MC-CDMA signal is capable of providing a near ideal PSD, which is flat over the system bandwidth available, while decreasing rapidly beyond that bandwidth. Explicitly, signals having this type of PSD are beneficial to both broadband and ultrawide-bandwidth communications. Furthermore, our results show that when optimum user address codes are employed, the single-user detector considered is near-far resistant, provided that the number of users supported by the system is lower than the number of subcarriers used for conveying an information symbol.
\end{abstract}

Index Terms-Code-division multiple access (CDMA), frequency-time matrix, multicarrier (MC), multiuser detection, Nakagami fading channels, power spectral density (PSD), timehopping (TH), ultrawide-bandwidth (UWB) radio.

\section{INTRODUCTION}

$\mathbf{T}$ IME-HOPPING (TH) is a type of spread-spectrum (SS) scheme, which was initially proposed in the 1960s (see [1], [2] and the references in them) in parallel with the other two types of SS schemes, namely direct-sequence SS (DS-SS) and frequency-hopping SS (FH-SS). However, over about three decades since its invention, the TH-SS scheme had received relatively less attention than the DS-SS and FH-SS schemes, until Scholtz proposed a TH-assisted pulse-positionmodulation (PPM) scheme for ultrawide-bandwidth (UWB) multiple-access communications [3], [4]. In TH-UWB communications [3], data is transmitted using extremely short timedomain pulses with duration of less than $1 \mathrm{~ns}$. Multiple users in UWB systems are usually supported with the aid of the TH techniques. Since UWB systems usually have a bandwidth on the order of gigahertz, a typical characteristic of UWB

Manuscript received April 18, 2004; revised October 19, 2005 and March 2, 2006. This work was supported in part by the financial assistance from EPSRC of U.K. and in part by the NEWCOM of the EU. The review of this paper was coordinated by Dr. A. Chockalingam.

The author is with the School of Electronics and Computer Science, University of Southampton, SO17 1BJ Southampton, U.K. (e-mail: 1ly@ecs. soton.ac.uk).

Digital Object Identifier 10.1109/TVT.2006.889577 channels is frequency-selective fading, which may result in a huge number of resolvable multipath components at the receiver [5]. Consequently, the receiver's complexity of the UWB systems might be extremely high, especially when carrier modulation is invoked, and when the receiver uses RAKElike coherent combining. This is because, in UWB, the RAKE receiver requires a very high number of fingers and each finger requires corresponding channel estimation, in order to achieve a high multipath combining efficiency.

In this paper, a time-hopping multicarrier code-division multiple-access (TH/MC-CDMA) scheme is proposed, where data is transmitted using relatively high-duration time-domain pulses with the aid of $M$-ary PPM (MPPM). At the receiver, information is extracted from one of the $M$ possible time-slot positions. In TH/MC-CDMA, each symbol is transmitted using a number of time-domain pulses with each time-domain pulse modulating a subcarrier. Since each subcarrier only occupies a fraction of the system bandwidth, the time-domain pulses, hence, have a relatively high duration. Consequently, even the total system bandwidth is extremely high, the subcarrier signals may be designed so that they are not too dispersive and only a low number of independent multipath components appear at the receiver. In TH/MC-CDMA system, diversity gain may be achieved by combining the independently faded multipath components or/and by transmitting the same information symbol using several subcarriers, which are combined at the receiver. In TH/MC-CDMA, the transmitter does not conflict the problem of nonlinear fluctuation resulted from the high peak-to-average factor [6], since, in the TH/MC-CDMA transmitter, the TH patterns may be designed so that only one of the subcarrier signals is activated at any time instant. However, fast-Fourier transform (FFT)-assisted-MC demodulation technique can still be invoked for demodulating multiuser TH/MC-CDMA signals. In fact, in TH/MC-CDMA, single-user or multiuser detection can be carried out after a single FFT-MC demodulation device. In addition to the aforementioned advantages, the proposed TH/MC-CDMA may provide some other advantages, which are summarized in Section V associated with our conclusions.

In this paper, we investigate the design and performance issues of TH/MC-CDMA systems. Specifically, a synchronous TH/MC-CDMA communication scheme is considered, and the parameter design in the TH/MC-CDMA system is investigated. It can be shown that, by appropriately selecting the system parameters, the TH/MC-CDMA signal can appear a Gaussian noiselike flat power spectral density (PSD) over the system bandwidth available. Furthermore, the PSD's out-band sidelobes of TH/MC-CDMA signals decrease much more rapidly, in comparison to a corresponding single-carrier 


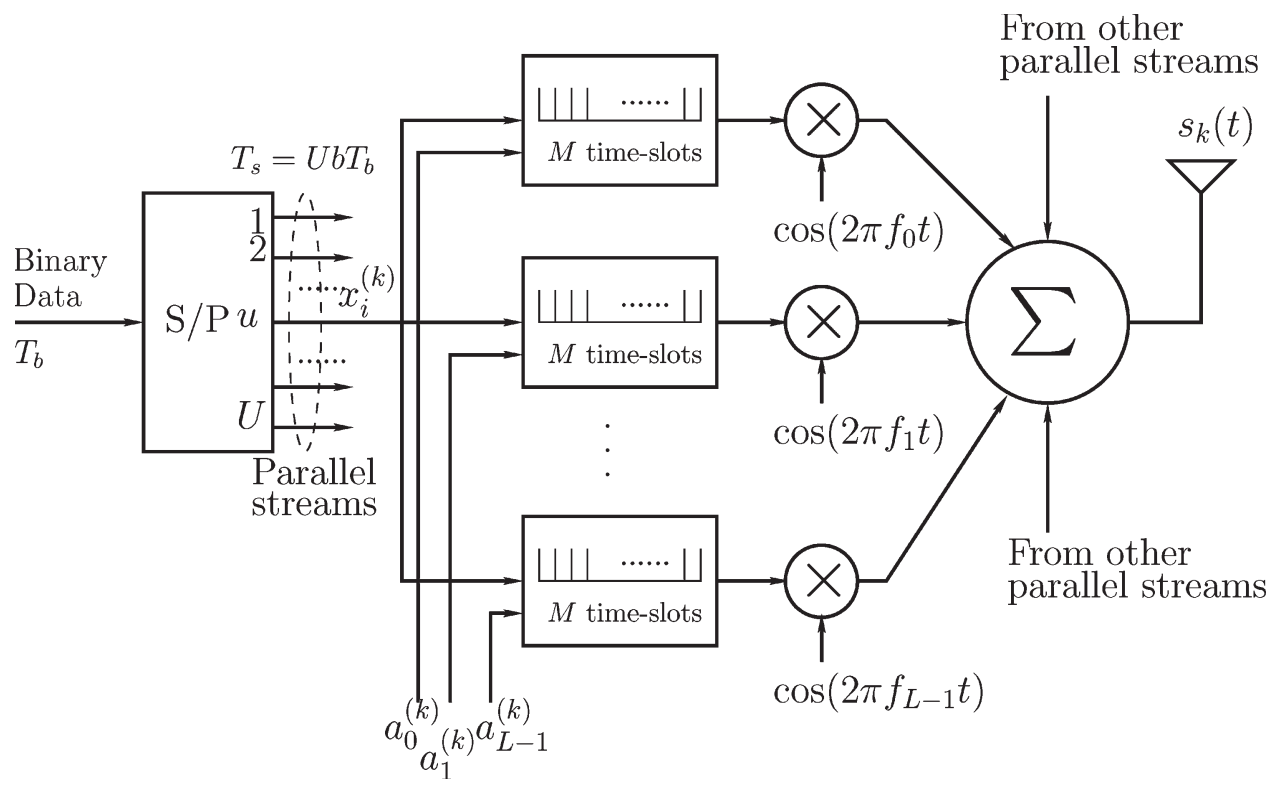

Fig. 1. $k$ th user's transmitter schematic for the TH/MC-CDMA.

TH-CDMA signals, which has the same system bandwidth as the TH/MC-CDMA signals. In this paper, a single-user detector is investigated, which is designed based on the frequency-timematrix principles. The bit-error-rate (BER) performance of the TH/MC-CDMA system is evaluated, when the subcarrier channels are modeled as flat Nakagami- $m$ fading in the presence of additive white Gaussian noise (AWGN).

The remainder of this paper is organized as follows. In the next section, the TH/MC-CDMA scheme is described and the parameters of this scheme are investigated. Furthermore, in this section, the PSD expression of the TH/MC-CDMA signal is considered. In Section III, we analyze a range of probabilities, including the probabilities of flag, null, and false alarm. In Section IV, the BER performance of the proposed TH/MCCDMA system is investigated using simulations. Finally, we provide our conclusions in Section $\mathrm{V}$ and indicate our further research topics.

\section{TH/MC-CDMA SCHEME}

\section{A. TH/MC-CDMA Signal}

The transmitter schematic of the $k$ th user is shown in Fig. 1 for the TH/MC-CDMA system. At the transmitter side, the input binary data having a period $T_{b}$ and a rate $R_{b}$ is serialto-parallel converted to $U$ parallel streams. During a frame interval of $T_{f}$ seconds, each stream transmits $b=\log _{2} M$ bits referred to as a symbol. Hence, each frame transmits $U$ symbols in parallel and both the symbol duration and the frame duration are $T_{s}=T_{f}=U b T_{b}$ seconds. Let the total number of subcarriers invoked in the TH/MC-CDMA system be $U L$; hence, each stream is transmitted on $L$ subcarriers. Let the TH address code of user $k, 1 \leq k \leq K$, be expressed as $\mathbf{a}_{k}=$ $\left[a_{0}^{(k)}, a_{1}^{(k)}, \ldots, a_{L-1}^{(k)}\right]$, where $a_{l}^{(k)} \in \mathrm{GF}(M), l=0,1, \ldots, L-$ 1 , and $\operatorname{GF}(M)$ denotes a Galois field obeying $M=2^{b}$. Furthermore, let $x_{k} \in \mathrm{GF}(M)$ be a $b$-bit symbol of one of the $U$ streams, say $u$, which will be transmitted by the $L$ subcarriers corresponding to the $u$ th stream. Then, $x_{k}$ is signatured by the $k$ th user's TH address code as

$$
\mathbf{Y}_{k}=\left[y_{k}(0), y_{k}(1), \ldots, y_{k}(L-1)\right]=x_{k} \cdot \mathbf{1} \oplus \mathbf{a}_{k}
$$

where 1 represents a unit vector of length $L, y_{k}(l), 0 \leq l \leq$ $L-1$ are elements of $\operatorname{GF}(M)$, and $\oplus$ denotes the addition operation in $\operatorname{GF}(M)$. As shown in Fig. 1, for each subcarrier branch, there are $M=2^{b}$ time slots within a frame duration of $T_{f}$ seconds. After the signature operation of (1), one of the $M$ time slots corresponding to each subcarrier branch is then activated by one of the elements of $\mathbf{Y}_{k}$. Specifically, for the $u$ th stream transmitted by $L$ subcarriers, the $y_{k}(l)$ th time slot of the $l$ th subcarrier will be activated for transmission, while the other $(L-1)$ time slots of the $l$ th subcarrier cease transmission. After the above described $\mathrm{TH}$ operation, the $l$ th subcarrier branch modulates a subcarrier frequency $f_{l}$ for $l=0,1, \ldots, L-1$ within the active time slot. Finally, as shown in Fig. 1, the $U L$ subcarrier-modulated signals are added in order to form the transmitted signal. Specifically, for the $u$ th stream, the transmitted signal can be expressed as

$$
\begin{aligned}
s_{k}(t)=\sqrt{\frac{2 P M}{L}} \sum_{l=0}^{L-1} \psi_{T_{h}}( & \left.t-y_{k}(l) T_{h}\right) \\
& \times \cos \left(2 \pi\left(f_{c}+f_{l}\right) t+\phi_{l}^{(k)}\right)
\end{aligned}
$$

where $P M$ represents the transmitted power, the factor $M$ is due to the fact that only one out of $M$ time slots is activated, $f_{c}$ represents the carrier frequency, $\psi_{T_{h}}(t)$ represents a pulse waveform defined within $\left[0, T_{h}\right)$ and it is normalized so that $\int_{0}^{T_{h}} \psi_{T_{h}}^{2}(t) d t=T_{h}, T_{h}$ is the duration of a time slot, and 


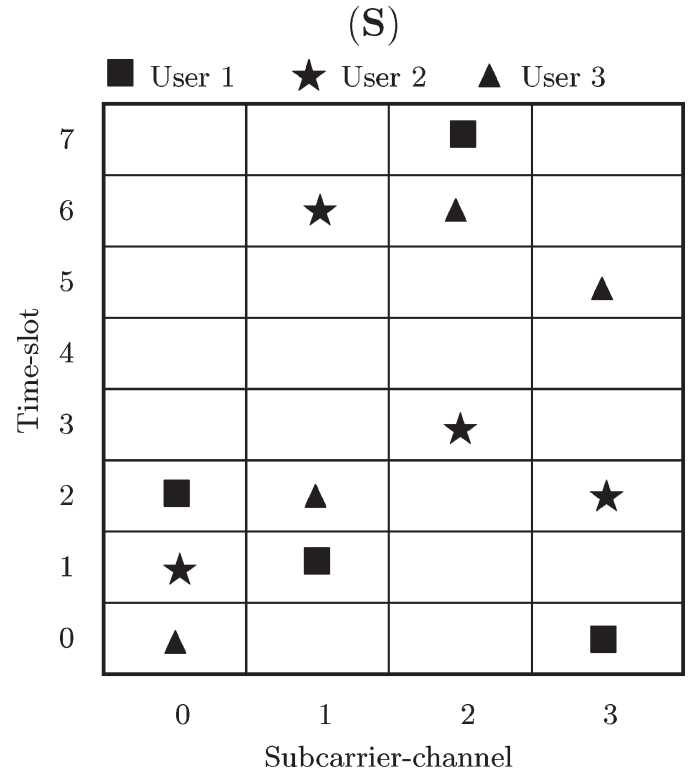

Fig. 2. Pictorial representation of transmitted signals for a three-user TH/MCCDMA system using $M=8$ time slots per symbol and $L=4$ subcarriers. The TH address codes are $[1,2,4,3]$ for user $1,[4,3,6,7]$ for user 2 , and $[7,5,1,2]$ for user 3, while the 3-bit symbols transmitted are $x_{1}=3$ for user $1, x_{2}=5$ for user 2 , and $x_{3}=7$ for user 3 .

finally, $\phi_{l}^{(k)}$ is the phase introduced by the TH. Note that (2) considers only one of the $U$ parallel streams. The other parallel streams can be considered in a similar way and have the same transmitted signal form as (2), except that the transmitted $b$-bit symbols and the subcarrier frequencies need to be changed correspondingly. However, for synchronous TH/MC-CDMA systems considered in this paper, the performance analysis can be carried out when considering only one of the parallel streams. Hence, unless otherwise mentioned, only one of the parallel streams is considered for the sake of simplicity in our forthcoming analysis.

\section{B. Modulation Parameters}

Let us assume a total $W_{s}$ hertz of bandwidth that the TH/MCCDMA system can exploit. Let us also assume that the input binary-data rate is $R_{b}=1 / T_{b}$. Below, we investigate the constraints associated with the parameters $M, L$, and $W_{\text {th }}$ of the TH bandwidth, which is defined as the null-to-null bandwidth per-subcarrier signal. The pictorial representation of the transmitted signal in a three-user TH/MC-CDMA system using $M=8$ time slots per frame and $L=4$ subcarriers is shown in Fig. 2, where we assumed that $x_{k}$ in (1) are $x_{1}=3, x_{2}=5$, and $x_{3}=7$, and the TH address codes are, respectively, [1, 2, 4, 3] for user $1,[4,3,6,7]$ for user 2 , and $[7,5,1,2]$ for user 3 .

First, when the TH duration is assumed to be $T_{h}$ seconds, then the frequency spacing between two adjacent TH tones should not be less than $R_{h}=1 / T_{h} \mathrm{~Hz}$, which is the constraint assumed in this paper. Consequently, for a TH/MC-CDMA system using $U L$ subcarriers, we have the relationship among the parameters $W_{s}, U$, and $L$ expressed as

$$
W_{s} \approx U L R_{h}=\frac{U L}{T_{h}} .
$$

Second, we have known that the frame duration is $T_{f}=$ $U b T_{b}$. Hence, the number of TH slots $M$ obeys

$$
M=2^{b}=\frac{T_{f}}{T_{h}}=\frac{U b T_{b}}{T_{h}} .
$$

Upon solving $T_{h}$ from (3) and substituting it into (4), we obtain

$$
\frac{M}{b}=\frac{T_{b} W_{s}}{L}=\frac{W_{s}}{R_{b} L} .
$$

As an example, let us assume that $W_{s}=4.096 \mathrm{MHz}, R_{b}=$ $32 \mathrm{~K} \mathrm{bit} / \mathrm{s}$, and $L=4$. Then, using (5), we have $2^{b} / b=32$, which can be satisfied when we select the number of bits per symbol to be $b=8$, implying that $M=2^{b}=256$. Furthermore, if the number of parallel streams or the number of symbols per frame is assumed to be $U=16$, then the TH rate can be derived from (3), which is $R_{h}=64 \mathrm{~K}$ hops/s.

\section{Power Spectral Density}

The PSD of the TH/MC-CDMA signals can be derived using an approach similar to those in [7] and [8]. Specifically, for real pulse-shape waveform of $\psi_{T_{h}}(t)$, the PSD can be expressed as

$S_{T}(f)=\frac{P M}{2 L T_{f}}\left\{\sum_{u=1}^{U} \sum_{m=0}^{L-1} \Psi^{2}\left(f-f_{u m}\right)+\sum_{u=1}^{U} \sum_{m=0}^{L-1} \Psi^{2}\left(f+f_{u m}\right)\right\}$

where $\Psi(f)$ represents the Fourier transform of the timedomain pulse $\psi_{T_{h}}(t)$, i.e., we have $\Psi(f)=\mathcal{F}\left\{\psi_{T_{h}}(t)\right\}$. Explicitly, (6) shows that the PSD of the TH/MC-CDMA signal is simply the sum of the basic pulse's PSD $\Psi^{2}(f)$ translated to all subcarrier frequencies and weighted by a constant of $P M / 2 L T_{f}$.

As an example, let us consider the $T_{h}$-width pulse $\psi_{T_{h}}(t)=$ $\sqrt{2} \cos \left(\pi t / T_{h}\right) \prod\left(t / T_{h}\right)$ [9], where $\prod\left(t / T_{h}\right)$ represents a rectangular waveform defined in $\left[0, T_{h}\right)$. The Fourier transform of $\psi_{T_{h}}(t)$ can be expressed as [9]

$$
\Psi(f)=\frac{2 \sqrt{2} T_{h}}{\pi} \times \frac{\cos \left(\pi T_{h} f\right)}{1-4 T_{h}^{2} f^{2}} .
$$

Upon substituting (7) into (6) and considering $T_{f}=M T_{h}$, the corresponding PSD can be expressed as

$$
\begin{aligned}
S_{T}(f)=\frac{4 P T_{h}}{L \pi^{2}}\left\{\sum_{u=1}^{U} \sum_{m=0}^{L-1} \frac{\cos ^{2}\left[\pi T_{h}\left(f-f_{u m}\right)\right]}{\left[1-4 T_{h}^{2}\left(f-f_{u m}\right)^{2}\right]^{2}}\right. \\
\left.+\sum_{u=1}^{U} \sum_{m=0}^{L-1} \frac{\cos ^{2}\left[\pi T_{h}\left(f+f_{u m}\right)\right]}{\left[1-4 T_{h}^{2}\left(f+f_{u m}\right)^{2}\right]^{2}}\right\} .
\end{aligned}
$$

The PSD of (8) for TH/MC-CDMA signals is shown in Fig. 3, when $U L=20$ subcarriers are employed. As shown in Fig. 3, the total system null-to-null bandwidth is about $W_{s}=$ $1.1 \mathrm{GHz}$ and the centered frequency is $f_{c}=3.025 \mathrm{GHz}$. Note that Fig. 3 only shows the PSD corresponding to the positivefrequency band, which were computed from the first summation of (8). According to (8), it is easy to know that the PSD corresponding to the negative-frequency band has the same 


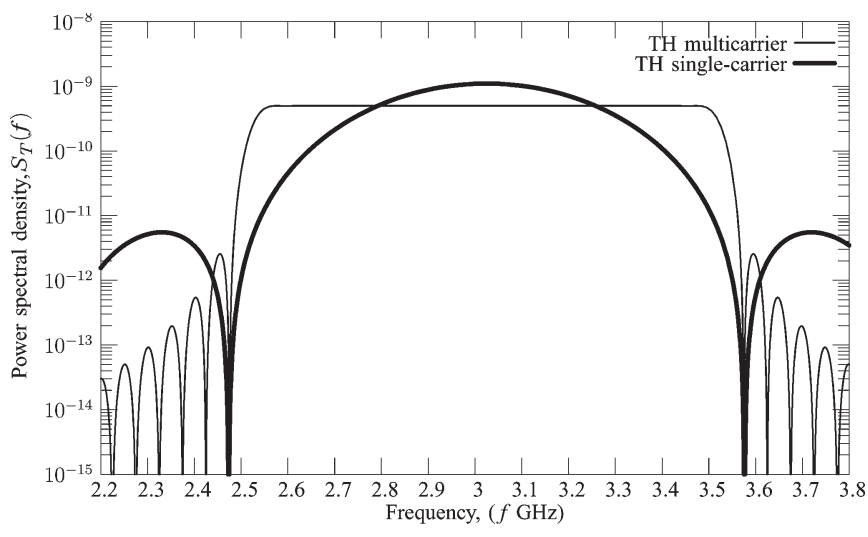

Fig. 3. PSD centered at $3.025 \mathrm{GHz}$ for both MC and single-carrier-based $\mathrm{TH}$ signals occupying a bandwidth of about $1.1 \mathrm{GHz}$, when 20 subcarriers of each having a bandwidth of $100 \mathrm{MHz}$ were assumed in TH/MC-CDMA scheme.

shape as that of the positive-frequency band but is centered at the frequency $f_{c}=-3.025 \mathrm{GHz}$. In Fig. 3, we also show the PSD of the single-carrier-based TH-CDMA signals, which occupies an equivalent bandwidth of $1.1 \mathrm{GHz}$. As shown in Fig. 3, TH/MC-CDMA signals have a flat Gaussian noiselike PSD over the available frequency band and the transmitted power is near-ideally distributed over the whole system bandwidth. This PSD characteristic is very important to broadband or UWB communications, since with the Gaussian noiselike PSD, the resource of bandwidth can be exploited with the highest efficiency. Furthermore, as shown in Fig. 3, the PSD's sidelobes of the TH/MC-CDMA signals decrease much more rapidly than that of the corresponding single-carrier TH-CDMA signals.

\section{Receiver Model}

We assume a free-space propagation channel with Nakagami- $m$ fading and AWGN. Consequently, when $K$ TH/MC-CDMA signals obeying the form of (2) are transmitted synchronously over this type of channels, the received complex low-pass equivalent signal can be expressed as

$$
\begin{aligned}
R(t)=\sum_{k=1}^{K} \sqrt{\frac{2 P M}{L}} \sum_{l=0}^{L-1} \alpha_{l}^{(k)} \psi_{T_{h}}\left(t-y_{k}(l) T_{h}\right) \\
\quad \times \exp \left(j\left[2 \pi f_{l} t+\varphi_{l}^{(k)}\right]\right)+N(t)
\end{aligned}
$$

where $N(t)$ represents the complex low-pass equivalent AWGN with zero-mean and single-sided PSD of $2 N_{0}$, while $\alpha_{l}^{(k)}$ represents an independent identically distributed (i.i.d.) Nakagami- $m$ random variable with a probability density function (PDF) given by [10] and [11]

$$
p_{\alpha_{l}^{(k)}}(R)=\frac{2 m^{m} R^{2 m-1}}{\Gamma(m) \Omega^{m}} e^{(-m / \Omega) R^{2}}
$$

where $\Gamma(\cdot)$ is the gamma function [12], $m$ is the Nakagami- $m$ fading parameter, and $\Omega=E\left[\left(\alpha_{l}^{(k)}\right)^{2}\right]$. Furthermore, in (9) $\varphi_{l}^{(k)}$, which takes into account of the phases due to both $\mathrm{TH}$ and channel fading, is assumed to be an i.i.d. random variable having a uniform distribution in $[0,2 \pi)$.
Due to the $\mathrm{TH}$ characteristic, we assume that the receiver is unable to estimate the fading amplitudes $\left\{\alpha_{l}^{(k)}\right\}$ and the phases $\left\{\varphi_{l}^{(k)}\right\}$, in addition to achieving synchronization with the received signal. The receiver schematic of the TH/MCCDMA system considered is shown in Fig. 4, which essentially represents an energy-assisted detector. The receiver constitutes a symbol-by-symbol or frame-by-frame detector. Specifically, the received signal is first MC demodulated with respect to each of the $L$ subcarriers used for the transmission of the $M=2^{b}$-ary symbol $x_{k}$. Then, the output signal for each subcarrier branch is input to a matched filter, which matches to the time-domain pulse $\psi_{T_{h}}(t)$ transmitted within a time slot. As shown in Fig. 4, the matched filter's output is sampled and, then, squared associated with each of the $M$ time slots in a frame, in order to generate a decision variable for detecting the corresponding energy level. Since there are $L$ subcarriers and each subcarrier conveys $M$ time slots, we are provided with a total of $M L$ outputs during a frame duration of $T_{f}=U b T_{b}$ s. As shown in Fig. 4, the time slots of $L$ subcarriers in the context of each frame are analyzed in order to determine, which time slots and, hence, which $b$-bit symbol $x_{k}$ was transmitted by invoking the detection scheme described below.

In TH/MC-CDMA systems, as described previously in the context of the transmitter of Fig. 1, a user address is employed as a unique signature sequence, in order to signal the time slots conveying a $b$-bit symbol of the user considered, as shown in (1). Following energy detection in terms of each time slot of the $L$ subcarriers, the sequence $\mathbf{Y}_{k}$ of (1) can be recovered by the receiver, provided that the channel is sufficiently good. By performing the subtraction operation of the unique user address $\mathbf{a}_{k}$ of the $k$ th user from $\mathbf{Y}_{k}$ on a subcarrier-by-subcarrier basis, we arrive at

$$
x_{k} \cdot \mathbf{1}=\mathbf{Y}_{k} \ominus \mathbf{a}_{k}
$$

which allows us to recover the transmitted symbol $x_{k}$ of user $k$. In (11), $\ominus$ represents the subtraction operation in $\mathrm{GF}(M)$ [13].

The detection operation of the TH/MC-CDMA system can be understood by means of frequency-time matrices ${ }^{1}$ having $M$ rows and $L$ columns, where the $M$ rows correspond to the $M$ distinct time slots of a frame, while the $L$ columns represent the $L$ subcarriers. We refer to the elements of the frequency-time matrices as frequency-time elements. The frequency-time matrix has the same feature as those shown in Fig. 2, where we assumed that a range of the frequency-time elements were activated by the transmitters of users 1,2 , and 3 . In order to make use of the frequency-time matrices for facilitating the detection at the receiver, the first step is to form a received frequency-time matrix $\mathbf{R}$ based on the decision variables. Specifically, let us assume that $h$ is a decision threshold. Then, whenever the energy-related decision variable, say $R_{l m}$ in Fig. 4, exceeds the threshold $h$, the corresponding frequency-time element at row $m$ column $l$ is flagged by a

\footnotetext{
${ }^{1}$ The frequency-time matrix described here is inspired by the concept of time-frequency matrix [14], which has been applied for describing the detection of fast-frequency-hopping (FFH) signals.
} 


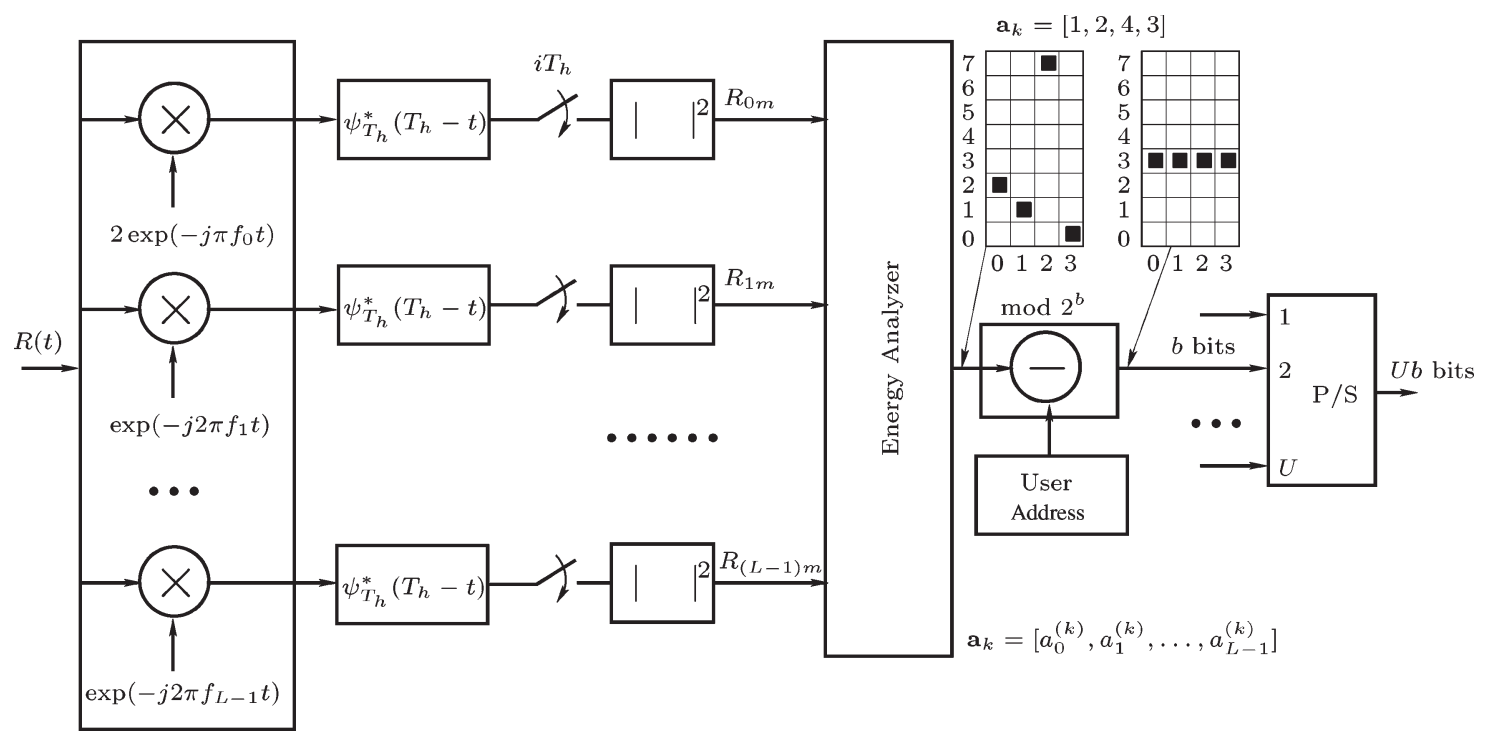

Fig. 4. Receiver block diagram of the TH/MC-CDMA scheme.

logical 1 (such as the markers in Fig. 2); otherwise, it is flagged by a logical 0 (null).

The received frequency-time matrix $\mathbf{R}$ considered in this paper has the same characteristics as that in [13]-[17]. Hence, various single-user- or multiuser-based detection schemes investigated in the aforementioned references can also be employed in a similar way in this paper for detecting the TH/MC-CDMA signals. In this paper, we consider only the single-user detector, when the transmitted TH/MC-CDMA signals experience Nakagami- $m$ fading. Furthermore, in this paper, we assume that optimum user addresses proposed by Einarsson in [13] are employed. Note that the analyses in [13]-[17] have been carried out by assuming noiseless channels having an infinity SNR and/or random user-signature addresses. The investigation concerning fading channels having limited SNR value as well as using optimum user-signature addresses is not available in the literature. Let us first give an example to show the detection principles.

Let the transmitted frequency-time matrix associated with three users be represented by Fig. 2. Provided that no channel impairments are imposed, all elements of the received frequency-time matrix, say $\mathbf{R}$, are received correctly, i.e., the received frequency-time matrix $\mathbf{R}$ is the same as the transmitted frequency-time matrix $\mathbf{S}$ of Fig. 2. Since the unique TH address assigned to user 1 is $\mathbf{a}_{1}=[1,2,4,3]$, the resultant frequency-time matrix $\mathbf{D}_{1}$ obtained by subtraction of the unique user address code $\mathbf{a}_{1}$ from $\mathbf{R}$ on a column-by-column basis according to (11) is shown in Fig. 5. Let us employ a simple majority-logic-decision (MLD)-based decoder, which opts for deciding the particular 8-ary symbol corresponding to the specific row having the highest number of nonzero entries, for the sake of providing an estimate to the transmitted symbol $x_{1}$ of user 1 . Hence, based on the frequency-time matrix $\mathbf{D}_{1}$ of Fig. 5, we infer that the transmitted symbol $x_{1}=3$ has been correctly decoded with the aid of unambiguous MLD. Furthermore, it can be shown that the transmitted symbols $x_{2}=5$ by user 2 and $x_{3}=7$ by user 3 can also be detected correctly by using the same approach described above.

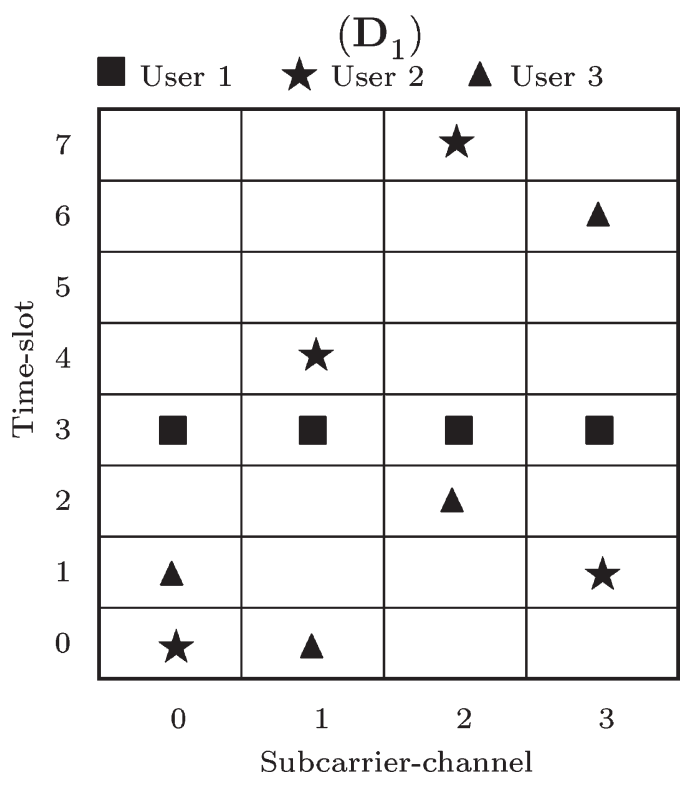

Fig. 5. Frequency-time matrix description for the detection of the 8-ary symbol $x_{1}=3$ transmitted by user 1 using the transmitted frequency-time matrix of Fig. 2. Explicitly, by processing the frequency-time matrix of Fig. 2 using the first user's address $\mathbf{a}_{1}=[1,2,4,3]$ according to (11) on a columnby-column basis, we obtain a unique complete row, which yields a unanimous symbol decision of $x_{1}=3$.

\section{STATistical ANALysis}

In this section, we analyze some statistics of the TH/MCCDMA system, which include the PDFs of the decision variables and the probabilities of flag, null, and false alarm.

\section{A. Decision Variable Statistics}

The output variable of Fig. 4 matched to the subcarrier $f_{l}$, and the $n$th time slot can be expressed as

$$
R_{l n}=\left|\int_{n T_{h}}^{(n+1) T_{h}} R(t) \psi_{T_{h}}^{*}(t) \exp \left(-j 2 \pi f_{l} t\right) d t\right|^{2}
$$


for $n=0,1, \ldots, M-1 ; l=0,1, \ldots, L-1$. Bearing in mind that orthogonal subcarriers are employed, then, upon substituting the received signal of (9) into the above equation, the decision variable can be expressed as

$$
\begin{gathered}
R_{l n}=\left|\sum_{k=1}^{K} \sqrt{\frac{2 E_{s} T_{h}}{L}} \alpha_{l}^{(k)} \exp \left(j \varphi_{l}^{(k)}\right) \delta\left[y_{k}(l), n\right]+N_{\text {oise }}\right|^{2} \\
n=0,1, \ldots, M-1 ; l=0,1, \ldots, L-1
\end{gathered}
$$

where $E_{s}=P T_{s}=P M T_{h}$ represents the transmitted energy per $M$-ary symbol, and the function $\delta[x, y]$ is defined by $\delta[x, y]=0$ for $x \neq y$ and $\delta[x, x]=1$. Furthermore, in (13), $N_{\text {oise }}$ contributed by $N(t)$ in (9) is a complex AWGN, both the real and imaginary parts have zero-mean and a common variance of $N_{0} T_{h}$.

For a given set of $\left\{\alpha_{l}^{(k)}\right\}$ and for a given set of $\left\{\varphi_{l}^{(k)}\right\}$, both the real part and the imaginary part of the random variable between the absolute-value sign of (13) are Gaussian random variables having a common variance of $N_{0} T_{h}$. Hence, the decision variable $R_{l n}$ represents a noncentral chi-square distribution [18] and the conditional PDF can be expressed as

$$
p_{R_{l n}}(z)=\frac{1}{2 N_{0} T_{h}} \exp \left(\frac{-\left(s^{2}+z\right)}{2 N_{0} T_{h}}\right) I_{0}\left(\sqrt{z} \frac{s}{N_{0} T_{h}}\right)
$$

where, by definition

$$
\begin{aligned}
s^{2}= & \left(\sum_{q=1}^{Q} \sqrt{\frac{2 E_{s} T_{h}}{L}} \alpha_{l}^{(q)} \cos \varphi_{l}^{(q)}\right)^{2} \\
& +\left(\sum_{q=1}^{Q} \sqrt{\frac{2 E_{s} T_{h}}{L}} \alpha_{l}^{(q)} \sin \varphi_{l}^{(q)}\right)^{2} \\
= & \frac{2 E_{s} T_{h}}{L}\left[\left(\sum_{q=1}^{Q} \alpha_{l}^{(q)} \cos \varphi_{l}^{(q)}\right)^{2}+\left(\sum_{q=1}^{Q} \alpha_{l}^{(q)} \sin \varphi_{l}^{(q)}\right)^{2}\right]
\end{aligned}
$$

where we assumed that there were $Q, Q=0,1,2, \ldots, K$ users, who share at the same time the $n$th time slot of the $l$ th subcarrier. Upon substituting (16) into (14) and letting $\mathcal{R}=$ $R_{l n} / N_{0} T_{h}$, the PDF of $\mathcal{R}$ can be expressed as

$$
p_{\mathcal{R}}(y \mid \gamma)=\frac{1}{2} \exp \left(-\frac{1}{2}[\gamma+y]\right) I_{0}(\sqrt{\gamma y}), \quad y \geq 0
$$

where the SNR of $\gamma$ is a function of the random variables $\left\{\alpha_{l}^{(q)}\right\}$ and $\left\{\varphi_{l}^{(q)}\right\}$, which is given by

$$
\gamma=\gamma_{c}\left[\left(\sum_{q=1}^{Q} \alpha_{l}^{(q)} \cos \varphi_{l}^{(q)}\right)^{2}+\left(\sum_{q=1}^{Q} \alpha_{l}^{(q)} \sin \varphi_{l}^{(q)}\right)^{2}\right]
$$

associated with $\gamma_{c}=2 E_{s} / L N_{0}$. Furthermore, in (17), $I_{0}(x)$ is the zeroth-order modified-Bessel function of the first kind defined in [12].

\section{B. Probabilities of Flag, False Alarm, and Null}

The probabilities of flag, false alarm, and null are necessary for analyzing those systems applying threshold tests, such as the TH/MC-CDMA of this paper and the FFH schemes [13]-[16]. Let $h>0$ be a threshold set for making a decision, whether the current frequency-time element (time slot of a given subcarrier) is flagged. The probability of flag $P_{G}$ is defined as the probability of the event that the decision variable corresponding to a frequency-time element exceeds the threshold $h$, which can be expressed as

$$
P_{G}(\gamma)=\int_{h}^{\infty} p_{\mathcal{R}}(y \mid \gamma) d y
$$

Assuming that there are $Q \geq 1$ users sharing a given frequency-time element, then, the corresponding average flagging probability for a given value of $Q \geq 1$ is given by

$$
\begin{aligned}
P_{G}(Q) & =\int_{0}^{\infty} P_{G}(\gamma) f(\gamma) d \gamma=\int_{0}^{\infty} \int_{h}^{\infty} p_{\mathcal{R}}(y \mid \gamma) f(\gamma) d y d \gamma \\
& =\int_{h}^{\infty} d y \int_{0}^{\infty} p_{\mathcal{R}}(y \mid \gamma) f(\gamma) d \gamma=\int_{h}^{\infty} p_{\mathcal{R}}(y) d y
\end{aligned}
$$

where $f(\gamma)$ represents the PDF of the SNR $\gamma$, which is expressed in (18), while

$$
p_{\mathcal{R}}(y)=\int_{0}^{\infty} p_{\mathcal{R}}(y \mid \gamma) f(\gamma) d \gamma
$$

represents the unconditional PDF of the variable $\mathcal{R}$. As shown in (18), $\gamma$ is a function of $\left\{\alpha_{l}^{(q)}\right\}$ and $\left\{\varphi_{l}^{(q)}\right\}$. Due to the inclusion of the sinusoid waveforms, it can be found that, except for some special cases, it is not straightforward to derive the exact analytical PDF $f(\gamma)$, when $Q>1$. Hence, it is not straightforward to derive the exact PDF of $p_{\mathcal{R}}(y)$ in (21) as well as the flag probability $P_{G}(Q)$ in (20) for the case of $Q>1$. Below, we first consider the flag probabilities for some special cases. Then, the computation of the flag probability for the general case is investigated.

1) Case $1(Q=1)$ : For the case of $Q=1$, according to (18), we have

$$
\gamma=\gamma_{c} \alpha_{l}^{2}
$$

Since $\alpha_{l}$ is a random variable obeying the Nakagami- $m$ distribution of (10), it can be shown that the SNR $\gamma$ of (22) obeys the distribution having the PDF given by [19]

$$
f(\gamma)=\frac{1}{\Gamma(m)}\left(\frac{m}{\bar{\gamma}_{c}}\right)^{m} \gamma^{m-1} e^{-\left(m / \bar{\gamma}_{c}\right) \gamma}, \quad \gamma>0
$$

where $\bar{\gamma}_{c}=\Omega \gamma_{c}$. Substituting the above equation into (21), the unconditional PDF of $\mathcal{R}$ can be obtained from (15) of [20] by 
correspondingly setting $U=1, \eta=0$, which yields

$$
\begin{aligned}
p_{\mathcal{R}}(y)=\frac{1}{2}\left(\frac{2 m}{2 m+\bar{\gamma}_{c}}\right)^{m} \exp \left(-\frac{m y}{2 m+\bar{\gamma}_{c}}\right) \\
\times{ }_{1} F_{1}\left(1-m, 1,-\frac{\bar{\gamma}_{c} y}{4 m+2 \bar{\gamma}_{c}}\right)
\end{aligned}
$$

where ${ }_{1} F_{1}()$ is the confluent hypergeometric function, which is defined as ${ }_{1} F_{1}(a, b, x)=\sum_{k=0}^{\infty}\left((a)_{k} x^{k}\right) /\left((b)_{k} k !\right), b \neq 0$, $-1,-2, \ldots, \quad$ and $\quad(a)_{k}=a(a+1)(a+2) \cdots(a+k-1)$, $(a)_{0}=1$ [12, pp. 504]. Finally, upon substituting (24) into (20), we can obtain the flag probability, which can be expressed as

$$
\begin{aligned}
P_{G}(Q=1)= & \left(\frac{2 m}{2 m+\bar{\gamma}_{c}}\right)^{m-1} \exp \left(-\frac{m h}{2 m+\bar{\gamma}_{c}}\right) \\
& \times \sum_{n=0}^{\infty} \sum_{k=0}^{n}\left(-\frac{\bar{\gamma}_{c}}{2 m}\right)^{n} \\
& \times\left(\frac{m}{2 m+\bar{\gamma}_{c}}\right)^{k} \frac{(1-m)_{n} h^{k}}{n ! k !} .
\end{aligned}
$$

2) Case $2(m=1)$ : We have known that the fading amplitudes $\left\{\alpha_{l}^{(q)}\right\}$ in (18) obey the Rayleigh distribution, when $m=1$. Hence, each term of $\alpha_{l}^{(q)} \cos \varphi_{l}^{(q)}$ or $\alpha_{l}^{(q)} \sin \varphi_{l}^{(q)}$ in (18) obeys the independent Gaussian distribution associated with mean of zero and a variance of $E\left[\left(\alpha_{l}^{(q)}\right)^{2}\right] / 2=\Omega / 2$. Consequently, both $\sum_{q=1}^{Q} \alpha_{l}^{(q)} \cos \varphi_{l}^{(q)}$ and $\sum_{q=1}^{Q} \alpha_{l}^{(q)} \sin \varphi_{l}^{(q)}$ obey the Gaussian distributions with zero-mean and a common variance of $Q \Omega / 2$. Hence, it is easy to show that the SNR $\gamma$ of (18) obeys the exponential distribution with the PDF expressed as

$$
f(\gamma)=\frac{1}{Q \bar{\gamma}_{c}} e^{-\gamma / Q \bar{\gamma}_{c}}, \quad \gamma>0
$$

where, again, $\bar{\gamma}_{c}=\Omega \gamma_{c}$. Substituting (17) and (26) into (21), it can be shown that, for this case, the unconditional PDF of $\mathcal{R}$ can be expressed as

$$
p_{\mathcal{R}}(y)=\frac{1}{2+Q \bar{\gamma}_{c}} \exp \left(-\frac{y}{2+Q \bar{\gamma}_{c}}\right) .
$$

Finally, upon substituting (27) into (20), the flag probability for the case of $m=1$ can be expressed as

$$
P_{G}(Q \mid m=1)=\exp \left(-\frac{h}{2+Q \bar{\gamma}_{c}}\right) .
$$

3) Case 3 (General): For the general case of an arbitrary $Q$ users sharing the same frequency-time element and communicating over Nakagami- $m$ fading channels, first, we notice that both $\sum_{q=1}^{Q} \alpha_{l}^{(q)} \cos \varphi_{l}^{(q)}$ and $\sum_{q=1}^{Q} \alpha_{l}^{(q)} \sin \varphi_{l}^{(q)}$ consist of the sum of i.i.d. variables in the form of $\alpha_{l}^{(q)} \cos \varphi_{l}^{(q)}$ or $\alpha_{l}^{(q)} \sin \varphi_{l}^{(q)}$. Therefore, when the value $Q$ is sufficiently high, both $\sum_{q=1}^{Q} \alpha_{l}^{(q)} \cos \varphi_{l}^{(q)}$ and $\sum_{q=1}^{Q} \alpha_{l}^{(q)} \sin \varphi_{l}^{(q)}$ can be approximated by Gaussian variables having zero-mean and a common variance of $Q \Omega / 2$. Consequently, when $Q \gg 1$ and when $m$ is close to one, the general case can be approximately viewed as the case of $m=1$, and the flag probability can be approximated by (28).

However, when the value of $Q$ is low, such as $Q=2,3, \ldots$, and the value of $m \gg 1$, the practical flag probability may be significantly different from that computed using the Gaussian approximation. Below, the approach developed by Goldman [21] is employed for deriving the flag probability of the general case. We start from the decision variable of (13). Assuming $Q \geq 1$ users sharing the frequency-time element $(n, l)$, then, after the normalization of (13) using $N_{0} T_{h}$, the corresponding decision variable $\mathcal{R}=R_{l n} / N_{0} T_{h}$ can be expressed as

$$
\mathcal{R}=\left|\sum_{q=1}^{Q} \sqrt{\gamma_{c}} \alpha_{l}^{(q)} \exp \left(j \varphi_{l}^{(q)}\right)+n_{\text {oise }}\right|^{2}
$$

where $\gamma_{c}=2 E_{s} / L N_{0}$, while $n_{\text {oise }}=N_{\text {oise }} / \sqrt{N_{0} T_{h}}$ represents a complex AWGN, both its real and image parts have zero-mean and unity variance.

Let $\alpha_{q}=\sqrt{\gamma_{c}} \alpha_{l}^{(q)}$ and $\varphi_{q}=\varphi_{l}^{(q)}$. Since $\alpha_{l}^{(q)}$ obeys the Nakagami- $m$ distribution having the PDF of (10), it can be shown that $\alpha_{q}$ obeys the PDF

$$
p_{\alpha_{q}}(R)=\frac{2}{\Gamma(m)}\left(\frac{m}{\bar{\gamma}_{c}}\right)^{m} R^{2 m-1} e^{\left(-m / \bar{\gamma}_{c}\right) R^{2}}, \quad R \geq 0
$$

where $\bar{\gamma}_{c}=\Omega \gamma_{c}$. Furthermore, the $n$th moment of $\alpha_{q}$ is given by [18]

$$
E\left[\left(\alpha_{q}\right)^{n}\right]=\frac{\Gamma(m+n / 2)}{\Gamma(m)}\left(\frac{\bar{\gamma}_{c}}{m}\right)^{n / 2} .
$$

In [21], Goldman has investigated the statistical properties of an $n$-dimensional Gaussian random vector $\mathbf{N}$ plus $\mathbf{S}$ (the sum of $M$ vectors), say $\mathbf{X}_{1}, \mathbf{X}_{2}, \ldots, \mathbf{X}_{M}$, which have random amplitudes, and independent arbitrary orientations in $n$-dimensional space. In this paper, the problem we are attempting to solve is that of computing the PDF of $\mathcal{R}$, where $\mathcal{R}$ is expressed in (29) and the probability $P_{G}(Q)=\operatorname{Pr}(\mathcal{R}>h)$. According to (29), our problem can be formed as

$$
\begin{aligned}
& \mathbf{S}=\sum_{q=1}^{Q} \mathbf{X}_{q}=\left(\sum_{q=1}^{Q} \alpha_{q} \cos \varphi_{q}, \sum_{q=1}^{Q} \alpha_{q} \sin \varphi_{q}\right) \\
& \mathbf{N}=\left(n_{\text {oise }}^{R}, n_{\text {oise }}^{I}\right) \\
& \mathcal{R}=|\mathbf{S}+\mathbf{N}|^{2}
\end{aligned}
$$


where $n_{\text {oise }}^{R}$ and $n_{\text {oise }}^{I}$ represent the real and image parts of $n_{\text {oise }}$, respectively. Upon extending the (25) and (27) in [21] and remembering that, for our case, the corresponding parameters are $n=2, M=Q$, and $\sigma^{2}=1$, we obtain

$$
\begin{aligned}
p_{\mathcal{R}}(y)= & \frac{1}{2} \exp \left(-\frac{y}{2}\right) \sum_{i=0}^{\infty}(-1)^{i} \frac{L_{i}^{(0)}(y / 2) \mu_{Q}^{(2 i)}}{2^{i} i !}, \quad y \geq 0 \\
P_{G}(Q)= & \operatorname{Pr}(\mathcal{R}>h) \\
= & \operatorname{Pr}(\sqrt{\mathcal{R}}>\sqrt{h})=\exp \left(-\frac{h}{2}\right) \\
& \times\left[1-\frac{h}{2} \sum_{i=1}^{\infty}(-1)^{i} \frac{L_{i-1}^{(1)}(h / 2) \mu_{Q}^{(2 i)}}{2^{i} i i !}\right]
\end{aligned}
$$

where $L_{n}^{(\alpha)}(x)$ represents the Laguerre polynomials, which is defined as [12]

$$
L_{n}^{(\alpha)}(x)=\sum_{m=0}^{n}(-1)^{m}\left(\begin{array}{c}
n+\alpha \\
n-m
\end{array}\right) \frac{x^{m}}{m !} .
$$

In (35) and (36), $\mu_{Q}^{(2 i)} \equiv E\left[|\mathbf{S}|^{2 i}\right]$ can be determined by the recurrence relations [21]

$$
\mu_{j}^{(2 i)}=\sum_{k=0}^{i}\left(\begin{array}{l}
i \\
k
\end{array}\right)^{2} \mu_{j-1}^{(2 k)} v_{j}^{(2 i-2 k)}
$$

for $j=2,3, \ldots, Q$. For the Nakagami- $m$ distributed variables $\left\{\alpha_{q}\right\}$, we have the following initial values for the above recurrence equation:

$$
\begin{aligned}
\mu_{1}^{(2 k)} & =E\left[\left|\mathbf{X}_{1}\right|^{2}\right]=E\left[\left|\left(\alpha_{1} \cos \varphi_{1}, \alpha_{1} \sin \varphi_{1}\right)\right|^{2 k}\right] \\
& =E\left[\alpha_{1}^{2 k}\right]=\frac{\Gamma(m+k)}{\Gamma(m)}\left(\frac{\bar{\gamma}_{c}}{m}\right)^{k} \\
v_{q}^{(2 k)} & =E\left[\left|\mathbf{X}_{q}\right|^{2}\right]=E\left[\left|\left(\alpha_{q} \cos \varphi_{q}, \alpha_{q} \sin \varphi_{q}\right)\right|^{2 k}\right] \\
& =E\left[\alpha_{q}^{2 k}\right]=\frac{\Gamma(m+k)}{\Gamma(m)}\left(\frac{\bar{\gamma}_{c}}{m}\right)^{k}, \quad q=2,3, \ldots, Q .
\end{aligned}
$$

Furthermore, the Laguerre polynomials $L_{i}^{(\alpha)}(x)$ in (35) and (36) can be expressed in terms of the Confluent Hypergeometric function ${ }_{1} F_{1}(\cdot, \cdot, \cdot)$ using the relation $[12, \mathrm{pp} .780]$

$$
L_{i}^{\alpha}(x)=\left(\begin{array}{c}
i+\alpha \\
i
\end{array}\right){ }_{1} F_{1}(-i, \alpha+1, x) .
$$

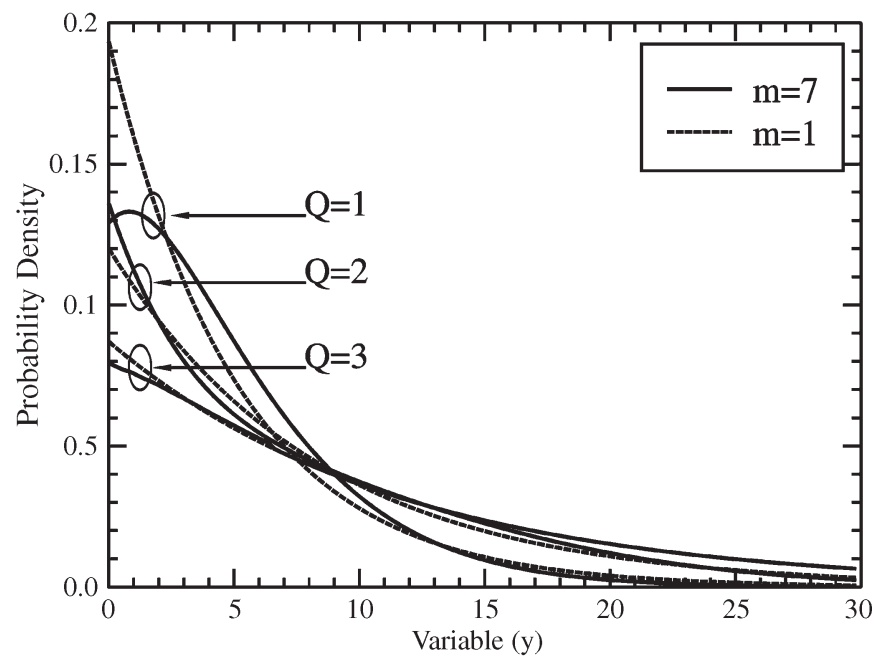

Fig. 6. PDF of the normalized decision variable $\mathcal{R}$ for the general case of (35) or (42) associated with the fading parameter of $m=7$ and the $m=1$ case of (27), where the $m=1$ case represents the Gaussian approximation of the general case.

Hence, (35) and (36) can also be expressed as

$$
p_{\mathcal{R}}(y)=\frac{1}{2} \exp \left(-\frac{y}{2}\right) \sum_{i=0}^{\infty}(-1)^{i} \frac{{ }_{1} F_{1}(-i, 1, y / 2) \mu_{Q}^{(2 i)}}{2^{i} i !}, \quad y \geq 0
$$

$$
\begin{aligned}
P_{G}(Q)= & \exp \left(-\frac{h}{2}\right) \\
& \times\left[1-\frac{h}{2} \sum_{i=1}^{\infty}(-1)^{i} \frac{(i+1){ }_{1} F_{1}(-i, 2, h / 2) \mu_{Q}^{(2 i)}}{2^{i} i i !}\right] .
\end{aligned}
$$

The PDF of (35) or (42) associated with the Nakagami- $m$ fading parameter $m=7$ and the PDF of (27) corresponding to the case of Rayleigh fading are drawn in Fig. 6, when the number of users sharing the same frequency-time cell is $Q=1,2$, and 3 . Note again that (27) represents the Gaussian approximation of the general case when $m>1$. From Fig. 6, we observe that the PDF curve of $m=7$ becomes closer to the PDF curve of $m=1$, when the value of $Q$ increases. This observation implies that when the value of $Q$ is sufficiently high, Gaussian approximation of the random variable $(\mathbf{S}+\mathbf{N})$ in (34) may be invoked for deriving the flag probability. We note, furthermore, without verification that the Gaussian approximation becomes more accurate if $m$ is closer to one.

Above, we have discussed the computation of the flag probability when $Q \geq 1$ users activate a given frequency-time element. When a frequency-time element is not activated by any users, i.e., when $Q=0$, the corresponding flag probability in fact represents the false-alarm probability, which can be expressed as

$$
P_{G}(0)=P_{F}=\int_{h}^{\infty} p_{R_{l n}}(y \mid \gamma=0) d y
$$


where $p_{R_{l n}}(y \mid \gamma=0)$ is given by (17) conditioned on $\gamma=0$, which can be expressed as $p_{R_{l n}}(y \mid \gamma=0)=0.5 e^{-y / 2}, y \geq 0$. Hence, it is readily shown that

$$
P_{G}(0)=P_{F}=\exp \left(-\frac{h}{2}\right)
$$

Finally, the null probability of a frequency-time element, which is defined as the probability of the event that a frequency-time element is not flagged, can be expressed as

$$
\bar{P}_{G}(Q)=1-P_{G}(Q)
$$

where $P_{G}(Q)$ is given in (25), (28), (36), or (43), respectively, for $Q \neq 0$, while it is given in (45) for $Q=0$.

\section{Error Probability}

The difficulty of analyzing the error probability for the TH/MC-CDMA system is that the number of users sharing a given frequency-time element is a random variable, which ranges from zero to $K$ when assuming that $K$ users are supported. It can be shown that even the values of $M, L$, and $K$ are moderate, the number of combinations required for the errorprobability analysis might be extreme. Therefore, in this paper, the results in Section IV were mainly obtained by simulations. However, when the optimum user addresses [13] are employed, we can have some observations, which are analyzed as follows.

When the optimum user-address-assignment scheme is employed, the $k$ th user's unique TH address can be expressed as [13]

$$
\mathbf{a}_{k}=\left(\lambda_{k}, \lambda_{k} \beta, \lambda_{k} \beta^{2}, \ldots, \lambda_{k} \beta^{L-1}\right)
$$

where $\lambda_{k}$ is an element of $\operatorname{GF}(M)$ assigned to user $k$, and $\beta$ is a fixed primitive element of $\operatorname{GF}(M)$. Assuming that the number of time slots $M$ per subcarrier is higher than the number of subcarriers $L$ of conveying the same $M$-ary symbol. When optimum user addresses are employed, the TH/MC-CDMA scheme has the following properties [13]. 1) The maximum number of user address codes available is equal to $M$ and 2) two transmitted signals with different address codes will coincide at most once in a frame duration. Furthermore, based on the aforementioned properties resulted from using the optimum user address codes, we can readily prove the following observation.

Observation 1: Let us assume that the detection scheme described in Section II-D is employed for detecting the TH/MCCDMA signals. When without background noise, i.e., when the SNR per symbol $E_{s} / N_{0} \rightarrow \infty$, the transmitted symbol can always be correctly detected, provided that the number of users supported by the TH/MC-CDMA system does not exceed the number of subcarriers conveying an $M$-ary symbol, i.e., provided that $K \leq L$. The above observation is based on the fact that there are no other completely flagged rows in the receiver frequency-time matrix, in addition to the complete row having $L$ entries flagged by the reference user. This is because, when using optimum user addresses, the maximum number of entries of an interfering row cannot exceed $(K-1) \leq(L-1)$.

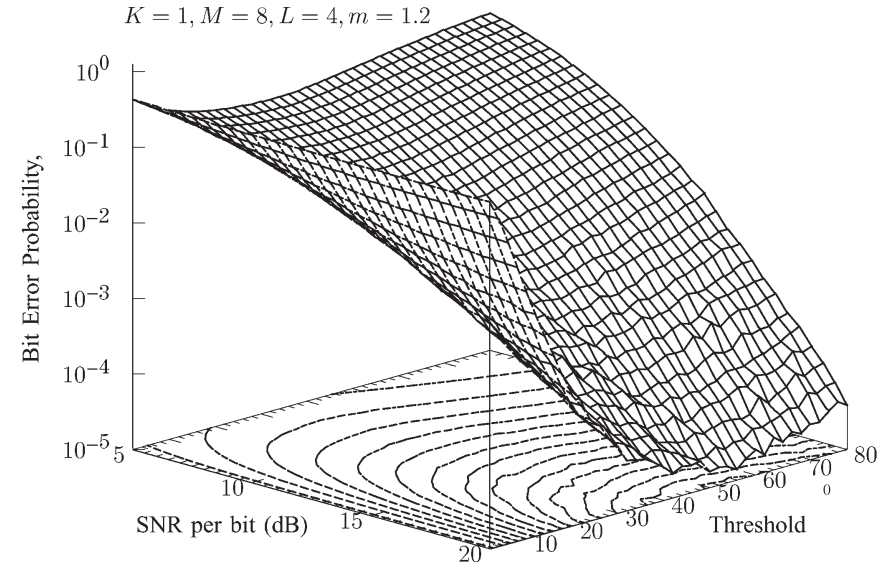

Fig. 7. Bit-error probability versus the SNR per bit $E_{b} / N_{0}$ and versus the threshold $h$ performance for the TH/MC-CDMA systems supporting $K=1$ user, when communicating over Nakagami- $m$ fading channels having the fading parameter $m=1.2$. The Galois field $\operatorname{GF}\left(M=8=2^{3}\right)$ corresponding to 3-bit symbols was used and that each 3-bit symbol was transmitted by $L=4$ subcarriers.

When noise exists in a TH/MC-CDMA system, we can be implied that the detection might be in error, even when the number of users supported by the TH/MC-CDMA is lower than the number of subcarriers conveying an $M$-ary symbol, i.e., when $K \leq L$. This is because the noise might generate false alarm or result in null. However, as shown in our results in Section IV, when there exists noise, the above observation can be stated: When noise exists, the TH/MC-CDMA signals are near-far resistance, provided that $K \leq L$. Let us now show the performance examples in the next section.

\section{Examples of Performance Results}

In this section, the performance of the TH/MC-CDMA systems using optimum user address codes is evaluated and compared for a range of parameters. Specifically, in our investigation, the optimum user address codes were generated according to (47) associated with using the Galois fields of $\mathrm{GF}(M=8), \mathrm{GF}(M=32)$ and $\mathrm{GF}(M=64)$. The corresponding primitive polynomials used for generating the user address codes were $p(x)=x^{3}+x+1$ for $\mathrm{GF}(8), p(x)=x^{5}+x^{2}+1$ for GF (32), and $p(x)=x^{6}+x+1$ for GF (64), respectively.

Fig. 7 shows the BER performance of the TH/MC-CDMA system versus the SNR per bit $E_{b} / N_{0}$ and versus the threshold $h$ when communicating over the Nakagami- $m$ fading channel having the fading parameter of $m=1.2$. The TH/MCCDMA system was assumed to support only a single user. It was also assumed that the Galois Field $\operatorname{GF}\left(8=2^{3}\right)$ corresponding to 3-bit symbols was used and that each 3-bit symbol was transmitted by $L=4$ subcarriers. It is clear from Fig. 7 that an inappropriate choice of the detection threshold $h$ may lead to significant increase of the BER. For any given threshold $h$, the BER decreases, as the SNR per bit increases. By contrast, for any given SNR per-bit value, there exists an optimal choice of the threshold $h$, which minimizes the BER. Furthermore, from Fig. 7, we observe that the optimum threshold value slightly increases as the SNR per-bit value increases. This property may 


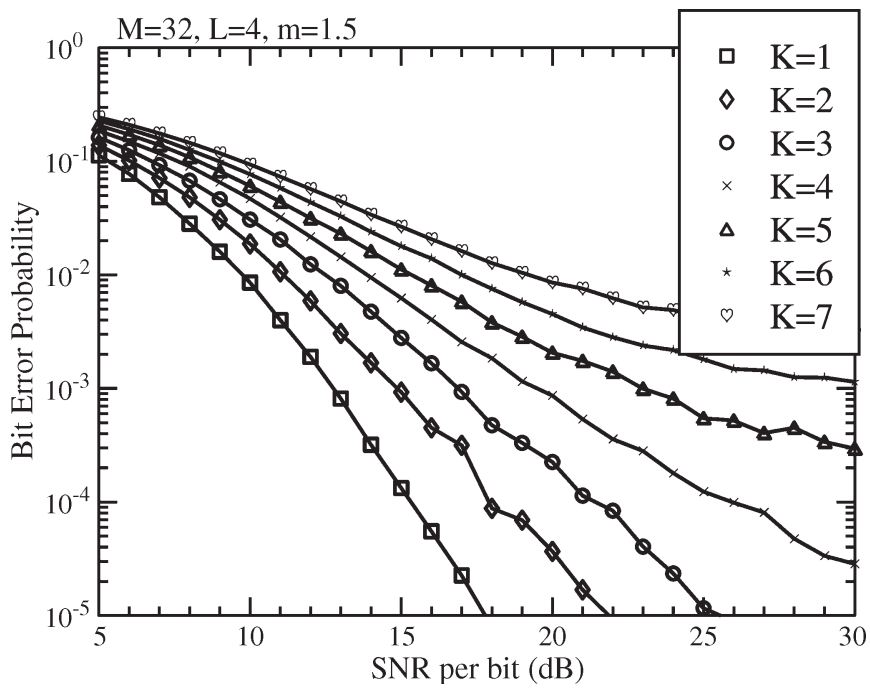

Fig. 8. BER versus SNR per-bit performance for the TH/MC-CDMA system using parameters of $M=32, L=4$ when communicating over Nakagami- $m$ fading channels having the fading parameter $m=1.5$.

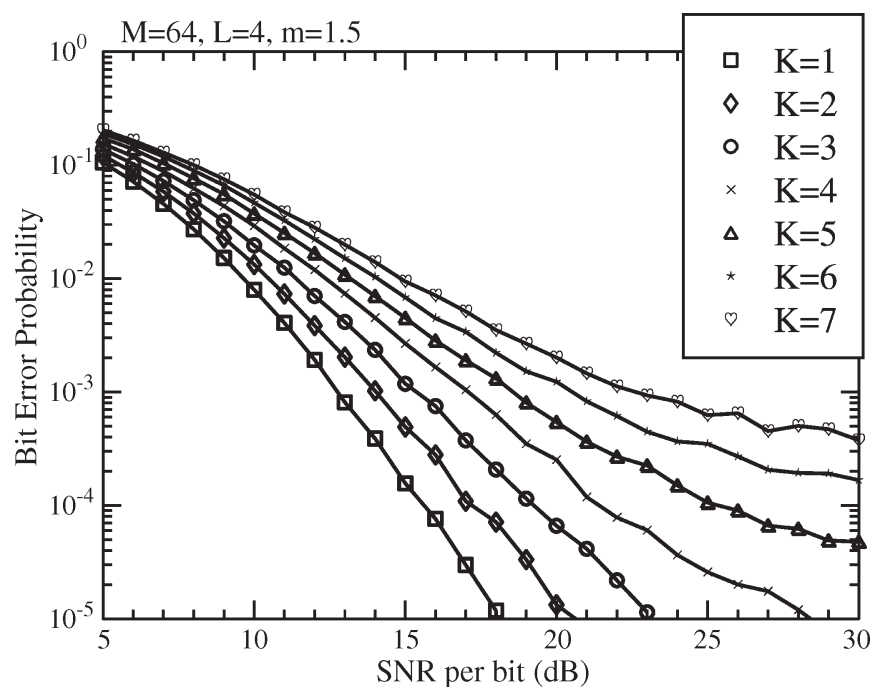

Fig. 9. BER versus SNR per-bit performance for the TH/MC-CDMA system using parameters of $M=64, L=4$ when communicating over Nakagami- $m$ fading channels having the fading parameter $m=1.5$.

be used for predicting the optimum thresholds at different SNR values. Hence, in our further investigations, we assumed that the optimum threshold was employed, whenever the detection threshold $h$ was invoked.

Figs. 8 and 9 show the bit-error probability of the TH/MCCDMA systems, when communicating over the Nakagami- $m$ fading channels associated with the fading parameter $m=1.5$. In our simulations of these figures, we assumed that each symbol was transmitted by $L=4$ subcarriers that the number of users supported were $K=1,2,3,4,5,6$, and 7 , and that the number of time slots per frame was $M=32$ for Fig. 8 and 64 for Fig. 9, respectively. From the results of Figs. 8 and 9, we observe that, for a given SNR per-bit value, the BER increases when the TH/MC-CDMA system supports more users or when the multiuser interference increases. It can also be seen that an interfering user imposes higher interference on the TH/MC-CDMA system having low $M$ value than on the system

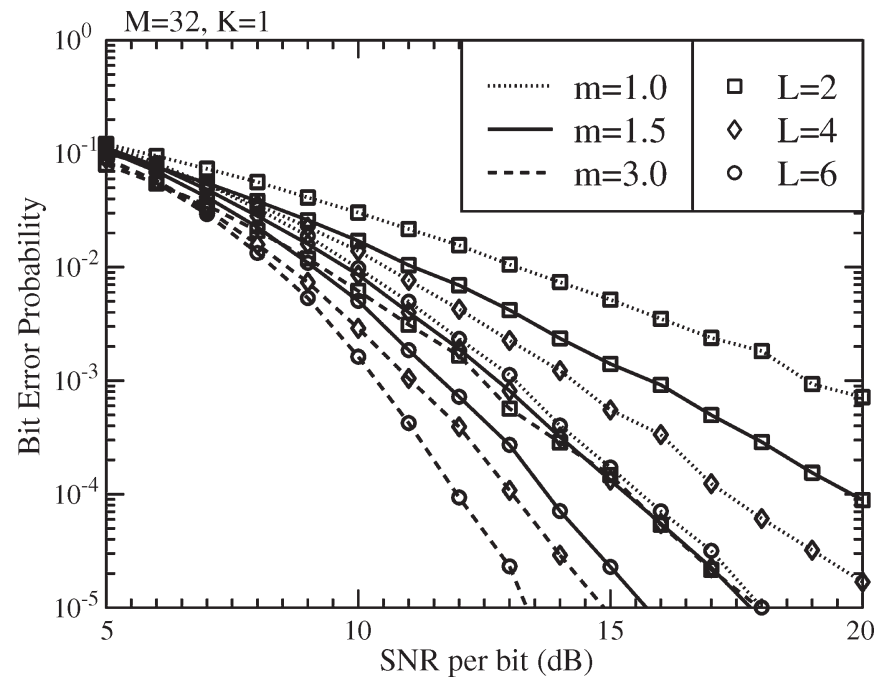

Fig. 10. BER versus SNR per-bit performance for the TH/MC-CDMA system using parameters of $M=32, K=1$ when communicating over Nakagami- $m$ fading channels with various fading parameters of $m=1,1.5$, and 3 and when each 5-bit symbol was transmitted by $L=2,4$, and 6 subcarriers, respectively.

having high $M$ value. The study in [13] and [17] has shown that when the number of users supported is not higher than the value of $L$, and when there is no background noise, the desired signal can always be decoded correctly, i.e., the desired signal can be decoded with BER of zero. When background noise exists, which is the case for both Figs. 8 and 9, we observe that if $K \leq L=4$ users is supported the BER decreases without error floor as the SNR per-bit value increases. By contrast, when the number of users supported is higher than $L=4$, we can clearly observe the error floors in these figures. Hence, for synchronous TH/MC-CDMA systems using optimum address codes, the considered single-user-based detector is near-far resistance, provided that the number of users supported by the system does not exceed the number of subcarriers conveying an $M$-ary symbol. Note that the error floors due to multiuser interference can be removed with the aid of multiuser detections [15]-[17], which constitutes our future research issues of the TH/MC-CDMA systems.

In Fig. 10, we show the influence of the fading parameter $m$ and the number of subcarriers $L$ on the bit-error probability. Note again that the case of $m=1$ corresponds to the Rayleigh fading channel while that the cases of $m=1.5,3$ represent the channels, such as Rician fading channels [10], that are less faded than the Rayleigh fading channels. From the results, we observe that for the TH/MC-CDMA scheme, assuming a given SNR per-bit value, the BER decreases when $m$ or $L$ increases. Furthermore, as shown in Fig. 10, the SNR gain corresponding to a low $m$ value, such as $m=1$, is more significant than that corresponding to a high $m$ value, such as $m=3$, when increasing the number of subcarriers $L$. For example, at the BER of $10^{-3}$, for $m=1$, the SNR gain is about $9.5 \mathrm{~dB}$ when we use $L=4$ subcarriers instead of $L=2$ subcarriers. By contrast, for the case of $m=3$, the SNR gain is only about $3 \mathrm{~dB}$ when increasing the number of subcarriers $L$ from two to four. Hence, in TH/MC-CDMA systems, when the wireless channels experience severe fading, a high number of subcarriers may be employed for conveying the same information symbol, in order 
to achieve a high-diversity gain, and, ultimately, to achieve a low bit-error probability.

\section{Conclusion}

We have proposed and investigated a novel multiple-access scheme, namely the TH/MC-CDMA scheme, which is based on the TH, MC modulation, and CDMA techniques. From our analysis and results, we find that TH/MC-CDMA is capable of providing a range of advantages. First, TH/MC-CDMA signals have a flat Gaussian noiselike PSD over probably a very wide bandwidth. The PSD outband sidelobes decrease much more rapidly than that of a corresponding single-carrier TH-CDMA signal. Second, in TH/MC-CDMA, due to the TH characteristic, each subcarrier is only activated for a fraction of the frame time duration. Hence, the nonlinear fluctuation due to the high peak-to-average factor in conventional MC systems can be significantly mitigated. Furthermore, in TH/MC-CDMA receiver, the processing rate is on the order of the TH rate $R_{h}$. For a given system bandwidth, the TH rate $R_{h}$ is inversely related to both the number of parallel data streams $U$ and the number of subcarriers $L$ conveying the same data symbol, i.e., $R_{h} \propto 1 / U L$. Hence, the detection is significantly less demanding than the demodulation during each chip interval, which would be necessary in DS-CDMA. In the TH/MC-CDMA systems supporting broadband or UWB communications, relatively low-rate parallel digital signal-processing techniques may be employed for implementing the transceivers. Additionally, the TH/MC-CDMA systems are in general less susceptible to the near-far problem than DS systems due to the TH characteristics. The multiuser detection and performance characterization in TH/MC-CDMA systems constitute the subject for future work.

\section{REFERENCES}

[1] J. H. Wittman, "Analysis of a hybrid frequency-time hopping randomaccess satellite communication system," IEEE Trans. Commun. Technol., vol. COM-16, no. 2, pp. 303-310, Apr. 1968.

[2] M. K. Simon, J. K. Omura, R. A. Scholtz, and B. K. Levitt, Spread Spectrum Communications: Volume I. Rockville, MD: Computer Science, 1985.

[3] R. A. Scholtz, "Multiple-access with time-hopping impulse modulation," in Proc. IEEE Mil. Commun. Conf., Oct. 1993, pp. 447-450.

[4] M. Z. Win and R. A. Scholtz, "Ultra-wide bandwidth time-hopping spread-spectrum impulse radio for wireless multiple-access communications," IEEE Trans. Commun., vol. 48, no. 4, pp. 679-691, Apr. 2000.

[5] F. Ramirez-Mireles, "On the performance of ultra-wide-band signals in Gaussian noise and dense multipath," IEEE Trans. Veh. Technol., vol. 50, no. 1, pp. 244-249, Jan. 2001.

[6] L. Hanzo, L.-L. Yang, E.-L. Kuan, and K. Yen, Single- and Multi-Carrier DS-CDMA. Hoboken, NJ: Wiley, 2003.

[7] M. Z. Win, "A unified spectral analysis of generalized time-hopping spread-spectrum signals in the presence of timing jitter," IEEE J. Sel. Areas Commun., vol. 20, no. 9, pp. 1664-1676, Dec. 2002.

[8] M. K. Simon, S. M. Hinedi, and W. C. Lindsey, Digital Communication Techniques—Signal Design and Detection. Englewood Cliffs, NJ: Prentice-Hall, 1995.
[9] R. E. Ziemer and R. L. Peterson, Digital Communications and Spread Spectrum Systems. New York: Macmillan, 1985.

[10] N. Nakagami, "The $m$-distribution, a general formula for intensity distribution of rapid fading," in Statistical Methods in Radio Wave Propagation, W. G. Hoffman, Ed. Oxford, U.K.: Pergamon, 1960.

[11] L.-L. Yang and L. Hanzo, "Performance of generalized multicarrier DS-CDMA over Nakagami-m fading channels," IEEE Trans. Commun. vol. 50, no. 6, pp. 956-966, Jun. 2002.

[12] M. Abramowitz and I. A. Stegun, Eds., Handbook of Mathematical Functions-With Formulas, Graphs, and Mathematical Tables. New York: Dover, 1972.

[13] G. Einarsson, "Address assignment for a time-frequency-coded, spreadspectrum system," Bell Syst. Tech. J., vol. 59, no. 7, pp. 1241-1255, Sep. 1980.

[14] D. J. Goodman, P. S. Henry, and V. K. Prabhu, "Frequency-hopped multilevel FSK for mobile radio," Bell Syst. Tech. J., vol. 59, no. 7 , pp. 1257-1275, Sep. 1980.

[15] K. W. Halford and M. Brandt-Pearce, "Multistage multiuser detection for FHMA," IEEE Trans. Commun., vol. 48, no. 9, pp. 1550-1562, Sep. 2000.

[16] U. C. G. Fiebig, "Iterative interference cancellation for FFH/MFSK MA systems," Proc. Inst. Electr. Eng.-Communications, vol. 143, no. 6 , pp. 380-388, Dec. 1996.

[17] L.-L. Yang and L. Hanzo, "Residue number system assisted fast frequency-hopped synchronous ultra-wideband spread-spectrum multiple-access: A design alternative to impulse radio," IEEE J. Sel. Areas Commun., vol. 20, no. 9, pp. 1652-1663, Dec. 2002.

[18] J. G. Proakis, Digital Communications, 3rd ed. New York: McGrawHill, 1995.

[19] L. L. Yang and L. Hanzo, "Low complexity erasure insertion in RS-coded SFH spread-spectrum communications with partial-band interference and Nakagami- $m$ fading," IEEE Trans. Commun., vol. 50, no. 6, pp. 914-925, Jun. 2002.

[20] L.-L. Yang and L. Hanzo, "Serial acquisition performance of singleand multi-carrier DS-CDMA over Nakagami- $m$ fading channels," IEEE Trans. Wireless Commun., vol. 1, no. 4, pp. 692-702, Oct. 2002.

[21] J. Goldman, "Statistical properties of a sum of sinusoids and Gaussian noise and its generalization to higher dimensions," Bell Syst. Tech. J., vol. 53, no. 4, pp. 557-580, Apr. 1974.

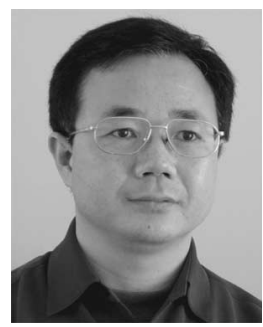

Lie-Liang Yang (M'98-SM'02) received the B.Eng. degree in communication engineering from Shanghai TieDao University, Shanghai, China, in 1988 and the M.Eng. and Ph.D. degrees in communications and electronics from Northern Jiaotong University, Beijing, China, in 1991 and 1997, respectively.

From June to December 1997, he was a Visiting Scientist of the Institute of Radio Engineering and Electronics, Academy of Sciences of the Czech Republic, Prague. Since December 1997, he has been with the Communications Research Group, School of Electronics and Computer Science, University of Southampton, Southampton, U.K., where he was first a Postdoctoral Research Fellow from December 1997 to August 2002 and a Lecturer from September 2002 to February 2006. Currently, he holds the academic post of Reader. His research has covered a wide range of areas in telecommunications, which include errorcontrol coding, modulation and demodulation, spread-spectrum communications and multiuser detection, synchronization, space-time processing, adaptive wireless systems, as well as wideband, broadband, and ultrawideband codedivision multiple-access. He has published over 130 papers in journals and conference proceedings, coauthored one book, and published several book chapters.

Dr. Yang is the recipient of the Royal Society Sino-British Fellowship in 1997 and the EPSRC Research Fellowship in 1998. He is currently an Associate Editor for both the Journal of Communications and Networks and the Journal of Communications. 